\title{
Position of diffuse satellites appearing in Ti-44Ni-6Fe shape memory alloy
}

\author{
Mitsuharu Todai $^{\mathrm{a}}$, Sho Majima ${ }^{1}$, Takashi Fukuda ${ }^{1}$, Tomoyuki Kakeshita ${ }^{1}$ \\ ${ }^{1}$ Department of Materials science and Engineering, Graduated School of Engineering, Osaka University, 2-1, \\ Yamada-oka, Suita, Osaka 565-0871, Japan
}

\begin{abstract}
We have investigated electron diffraction patterns of Ti-44Ni-6Fe alloy exhibiting a negative temperature dependence in electrical resistivity below $T_{\min }=210 \mathrm{~K}$. The electron diffraction patterns taken below $T_{\min }$ show diffuse satellites at $\mathbf{g}_{\mathrm{B} 2}+<\zeta \zeta 0>^{*}$ when the zone axis is [111] $]_{\mathrm{B} 2}$ and [100] $\mathrm{B} 2$. For both beam directions, the value of $\zeta$ is slightly smaller than $1 / 3$ and shows the same temperature dependence. On the other hand, the satellites are missing when the zone axis is [110] B2. This means that the incommensurate phase has a modulated structure with a propagation vector $\left\langle\zeta \zeta 0>^{*}(\zeta \sim 1 / 3)\right.$ and displacement direction is one of $<110>$ which is vertical to the propagation vector. This modulation is obviously related to the softening of $\mathrm{TA}_{2}$ phonon branch with the propagation vector near $1 / 3<\zeta \zeta 0>^{*}$. In addition to the satellites at $\mathbf{g}_{\mathrm{B} 2}+1 / 3<\zeta \zeta 0>^{*}$, satellites appear at $\mathbf{g}_{\mathrm{B} 2}+<\zeta \zeta 0>^{*}$ with $\zeta=0.5$ when the zone axis is $[100]_{\mathrm{B} 2}$ and rod-like steaks appear in $\left\langle\zeta \zeta 2 \zeta>^{*}\right.$ direction when the zone axis is [110] ${ }_{\mathrm{B} 2}$. However, these satellites and rod-like streaks do not show clear temperature dependence, suggesting that they are not directly related to the instability of the B2-type structure.
\end{abstract}

\section{Introduction}

The R-phase transformation in Ti-Ni based shape memory alloys is preceded by anomalies in physical properties, which are frequently referred to as precursor phenomena. These anomalies have attracted much attention because they are considered to be related to the origin of martensitic transformation. Phonon softening in $\mathrm{TA}_{2}$ branch $[1,2]$ and appearance of diffuse satellites [3-6] are representatives of such anomalies. In particular, many works have been made on diffuse satellites near $\mathbf{g}_{\mathrm{B} 2}+1 / 3<\zeta \zeta 0>^{*}$. Salamon and Wayman carried out experiments for a $\mathrm{Ti}_{50} \mathrm{Ni}_{46.8} \mathrm{Fe}_{3.2}$ single crustal and $\mathrm{Ti}_{50} \mathrm{Ni}_{47} \mathrm{Fe}_{3}$ polycrystals by a neutron diffraction, an $\mathrm{X}$-ray diffraction and an electron microscopy. As a result, they found that the diffuse satellites appear at an incommensurate position, and these reflections have temperature dependence [3]. They explained these satellites by the formation of charge density wave (CDW) [7]. On the other hand, Shapiro et al. made a study on an incommensurate state in single crystal by an X-ray diffraction and reported that the positions of diffuse satellites are not periodic. Thus, they concluded that the diffuse satellites cannot be explained by CDW [4]. In order to account for this peculiar incommensurability, Yamada suggested a modulated lattice relaxation (MLR) model [5]. Recently, Murakami and Shindo investigated the R-phase transformation in a $\mathrm{Ti}_{50} \mathrm{Ni}_{48} \mathrm{Fe}_{2}$ by in-situ electron microscopy equipped with an energy-filter [6]. They reported that the peak position and intensity of the diffuse satellites have temperature dependence and dark-field images with the diffuse satellites showed microdomains. Based on these results, they concluded that a microdomain is not a small R-phase but a single transverse atomic displacement. We also investigated this behavior and reported in the paper [8]. That is we investigated the composition and temperature dependences of the diffuse satellites appearing in electron diffraction patterns of Ti- $(50-x) \mathrm{Ni}-x \mathrm{Fe}$ alloys $(6 \leq x \leq 10)$, which exhibit a negative temperature coefficient of resistivity below a temperature $T_{\min }$ as shown in Fig. 1. Below $T_{\min }$, these alloys exhibit the diffuse satellites and the intensity maximum is located at $\left\langle\zeta \zeta 0>^{*}\right.$. The value of $\zeta$ increases with decreasing temperature, and decreases with increasing iron content. We also calculated the generalized susceptibility of the parent phase of these alloys from a first-principles calculation in order to know the existence of the nesting vector. Considering these results, we compared the scattering vector of diffuse satellites with a nesting vector and concluded that the incommensurate

\footnotetext{
a e-mail: mitsuharu.todai@mat.eng.osaka-u.ac.jp
}

This is an Open Access article distributed under the terms of the Creative Commons Attribution-Noncommercial License (http://creativecommons.org/licenses/by-nc/3.0/), which permits unrestricted use, distribution, and reproduction in any noncommercial medium, provided the original work is properly cited. 


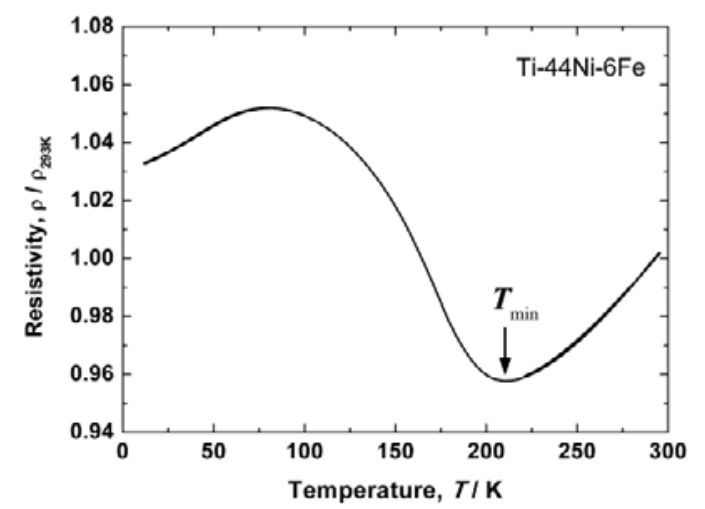

Fig. 1. Temperature dependence of electrical resistivity in Ti-44Ni-6Fe alloy.

phase (IC-phase) of Ti- $(50-x) \mathrm{Ni}-x \mathrm{Fe}$ alloys, which has diffuse satellites at incommensurate position, originates from the nesting effect of the Fermi surface with the B2-type structure [9].

In order to understand the structure of the IC-phase in more detail, we need to know the distribution of diffuse scatterings in reciprocal space. The purpose of the present study, therefore, is to clarify the direction and the temperature dependence of diffuse scatterings of IC-phase in Ti-44Ni-6Fe alloy by using a transmission electron microscope (TEM) with beam directions of [111] $\mathrm{B} 2,[100]_{\mathrm{B} 2}$ and $[110]_{\mathrm{B} 2}$.

\section{Experimental procedure}

An ingot of Ti-44Ni-6Fe alloy was prepared by an arc melting method and a single-crystalline rod was grown by a floating zone (FZ) method with a growth rate of $10 \mathrm{~mm} / \mathrm{h}$. The single-crystalline rod was homogenized at $1273 \mathrm{~K}$ for $24 \mathrm{~h}$ and heat-treated at $1273 \mathrm{~K}$ for $1 \mathrm{~h}$ to reduce internal strain, followed by quenching into iced water. Then the specimens were electropolished in an electrolyte composed of $95 \%$ acetic acid and $5 \%$ perchloric acid. TEM observations were made using a HITACHI H- 800 operated at $200 \mathrm{kV}$.

\section{Results and Discussion}

Figure 2(a), (b) and (c) show typical electron diffraction patterns of the Ti-44Ni-6Fe alloy taken at 190K. This temperature is $20 \mathrm{~K}$ below the temperature of resistivity minimum $\left(T_{\min }=210 \mathrm{~K}\right)$.

When the zone axis is [111] B2, the electron diffraction pattern shows diffuse satellites near $\mathbf{g}_{\mathrm{B} 2}+1 / 3<\zeta \zeta 0>^{*}$ in addition to the reflections of the B2-type structure as shown in Fig. 2(a). The detailed position of intensity maximum of diffuse satellites at $\mathbf{g}_{\mathrm{B} 2}+<\zeta \zeta 0>^{*}$ is investigated by the intensity profile scanned from the dotted frame in Fig. 2(a). The result is shown in Fig. 2(a'). It is noted in the figure that. The profile taken above $T_{\min }(300 \mathrm{~K}$ and $228 \mathrm{~K})$ shows only a peak of the B2-type structure. However, the profiles taken below $T_{\min }$ are composed of the B2-type structure and diffuse satellites near $\zeta=1 / 3$ and $2 / 3$. The intensity of diffuse satellites increase and the position gradually shift toward commensurate position of $\mathbf{g}_{\mathrm{B} 2}+1 / 3<\zeta \zeta 0>^{*}$ with decreasing temperature. These results are consistent with the previous study [8].

When the zone axis is [100] ${ }_{\mathrm{B} 2}$, the electron diffraction pattern taken at $190 \mathrm{~K}$ shows diffuse satellites near $\mathbf{g}_{\mathrm{B} 2}+1 / 3<\zeta \zeta 0>^{*}$ and at $\mathbf{g}_{\mathrm{B} 2}+1 / 2<\zeta \zeta 0>^{*}$ in addition to the B2-phase pattern as shown in Fig. 2(b). Figure 2(b') shows the one-dimensional intensity profile from the dotted frame in Fig 2(b). The profile taken above $T_{\min }$ $(300 \mathrm{~K}, 251 \mathrm{~K}$ and $232 \mathrm{~K})$ shows the peak at $\mathbf{g}_{\mathrm{B} 2}+1 / 2<\zeta \zeta 0>^{*}$ and the peak of the B2-type structure. Diffuse satellites near $\mathbf{g}_{\mathrm{B} 2}+1 / 3<\zeta \zeta 0>^{*}$ appear below $T_{\min }$ and they depend on temperature as observed in the [111] $\mathrm{B} 2$ zone axis. On the other hand the intensity and the position of $\mathbf{g}_{\mathrm{B} 2}+1 / 2<\zeta \zeta 0>^{*}$ are nearly independent of temperature.

When the zone axis is $[110]_{\mathrm{B} 2}$, the electron diffraction pattern taken at $190 \mathrm{~K}$ exhibits rod-like streaks along $<\zeta \zeta 2 \zeta>^{*}$ in addition to the reflections from the B2-type structure. Figure 2(c') shows the one-dimensional intensity profile from the dotted frame in Fig. 2(c). These profiles taken at all the temperature examined show only the peak of the B2-type structure at $\zeta=0$ and 1 . In order to know the direction of rod-like streaks, we have also investigated the intensity profile along $\left[, \zeta \zeta \zeta^{-}, \zeta\right]^{*}$ direction. As a results, the peak of the rod-like streaks exists in $\zeta \sim 0.62$ and the angle between streaks and $\left\langle\zeta \zeta 2 \zeta>^{*}\right.$ direction is about $5^{\circ}$ in reciprocal space. The rodlike streaks do not show clear temperature dependence. 

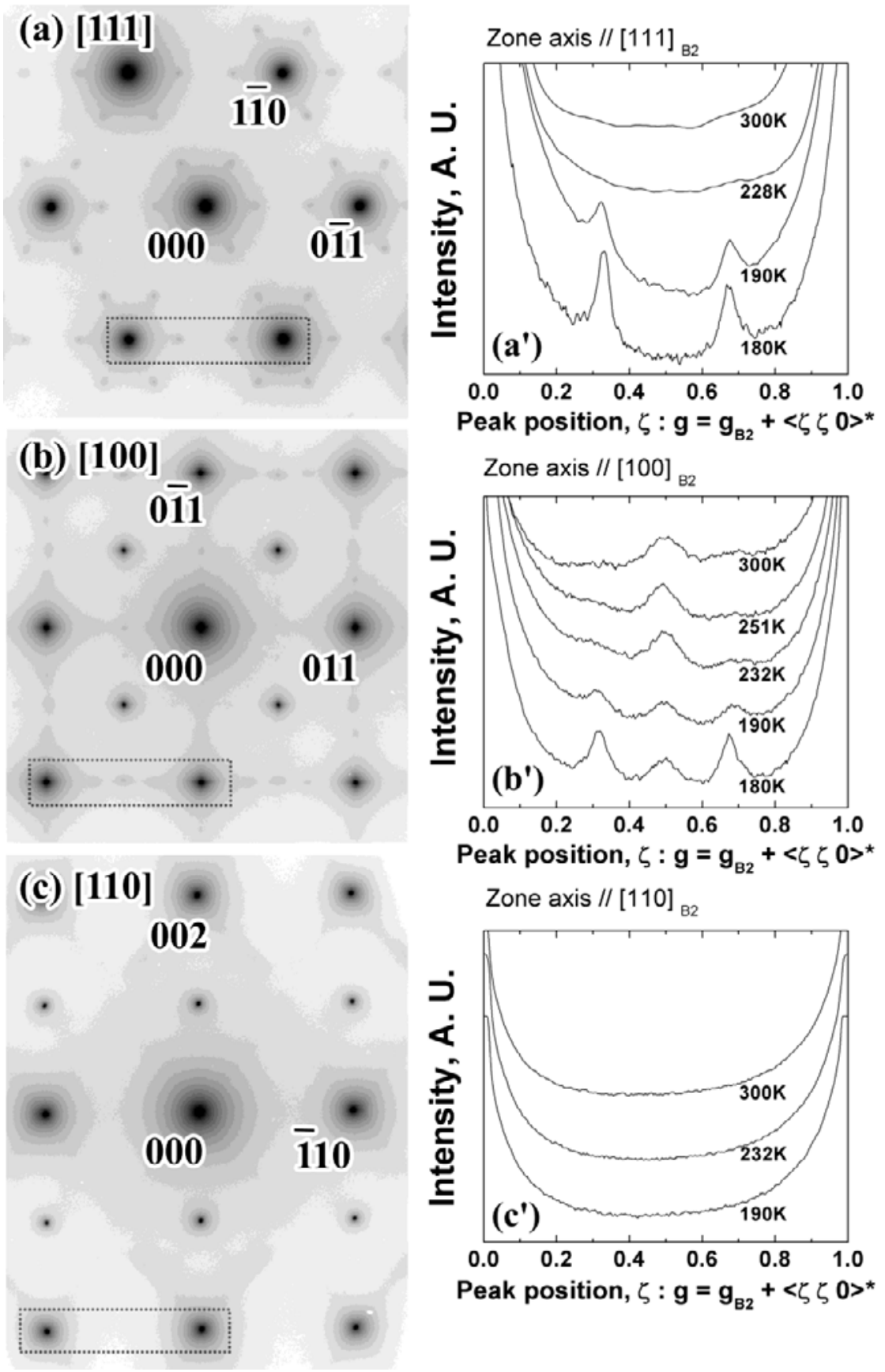

Fig. 2. Electron diffraction patterns of IC-phase in Ti-44Ni-6Fe alloy at $190 \mathrm{~K}$. The zone axis is $[111]_{\mathrm{B} 2}$ in (a), the zone axis is $[100]_{\mathrm{B} 2}$ in (b) and the zone axis is [110] ${ }_{\mathrm{B} 2}$ in (c) and intensity profile (a'), (b') and (c') from the dotted frame in (a), (b) and $(\mathrm{c})$, respectively.

As described above, the diffuse satellites appear near $\mathbf{g}_{\mathrm{B} 2}+1 / 3<\zeta \zeta 0>^{*}$ below $T_{\min }$ when the zone axis is [111] в2 and [100] в2. On the other hand, when the zone axis is [110] в2, the diffuse satellites near $\mathbf{g}_{\mathrm{B} 2}+1 / 3<\zeta \zeta 0>^{*}$ are missing. This means that the IC-phase has a modulated structure with the propagation vector $<\zeta \zeta 0>^{*}(\zeta \sim 1 / 3)$ and the displacement direction is vertical one of $<110>$ direction perpendicular to the propagation vector. This modulation is obviously related to the softening of $\mathrm{TA}_{2}$ phonon branch with the propagation vector near $1 / 3<\zeta \zeta 0>^{*}$. In addition, we compare the temperature dependence of the diffuse satellites observed in the diffraction patterns of $[111]_{\mathrm{B} 2}$ zone axis and that observed in the diffraction patterns of [100] $\mathrm{B} 2$ zone axis. The result is shown in Fig. 3, where the position parameter $\zeta$ of the satellites obtained from the line 


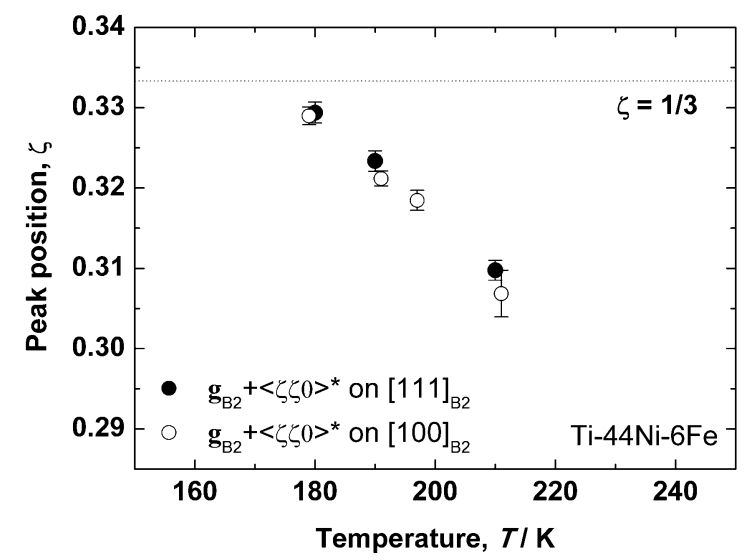

Fig. 3. Peak position $\zeta$ of intensity maximum of diffuse satellites $\left(\mathbf{g}_{\mathrm{B} 2}+<\zeta \zeta 0>^{*}\right)$ in electron diffraction patterns plotted as a functional of temperature in Ti-44Ni-6Fe alloy. Dotted line corresponds $\zeta=1 / 3$.

profile shown in Fig. 2(b') and (c'). We notice that there is not significant difference in the temperature dependence of $\zeta$ for the two zone axes. This means that the positions of diffuse satellites appear periodically in the precision of electron diffraction. Such a periodic nature of the satellites of IC-phase is consistent with the interpretation that the origin of IC-phase is nesting effect of the Fermi surface with the B2-type structure.

Finally, the diffuse satellites at $\mathbf{g}_{\mathrm{B} 2}+1 / 2<\zeta \zeta 0>^{*}$ as observed in the [100] B2 zone axis and rod-like streaks as observed in the [110] в2 do not have temperature dependence. Possibly, they are not related to the instability of the B2-type structure.

\section{Conclusions}

We have investigated the structure of the IC-phase by electron diffraction experiments with zone axes of $[111]_{\mathrm{B} 2}$ $[100]_{\mathrm{B} 2}$ and $[110]_{\mathrm{B} 2}$. As a result, diffuse satellites appear at $\mathbf{g}_{\mathrm{B} 2}+<\zeta \zeta 0>^{*}(\zeta \sim 1 / 3)$ when the zone axis is $[111]_{\mathrm{B} 2}$ and $[100]_{\mathrm{B} 2}$. The value of $\zeta$ is almost the same for the two beam direction, for all the temperatures examined. On the other hand, the satellites at $\mathbf{g}_{\mathrm{B} 2}+<\zeta \zeta 0>^{*}(\zeta \sim 1 / 3)$ is missing when the zone axis is [110] B2. Consequently, it is concluded that the incommensurate phase has a modulated structure with propagation vector $<\zeta \zeta 0>^{*}(\zeta \sim 1 / 3)$ and the displacement direction is one of $<110>$ vertical to the propagation vector. Satellites also appear at $\mathbf{g}_{\mathrm{B} 2}+<\zeta \zeta 0>^{*}$ with $\zeta=0.5$ when the zone axis is [100] ${ }_{\mathrm{B} 2}$ and rod-like steaks appear in $<\zeta \zeta 2 \zeta>^{*}$ direction when the zone axis is $[110]_{\mathrm{B} 2}$, but they are not directly related to the instability of the B2-type structure.

\section{Acknowledgments}

This work was partly supported by the Formation of Worldwide Renowned Centers of Research-The Global Century COE Program (Project: Center of Excellence for Advanced Structural and Functional Materials Design) from the Ministry of Education, Culture, Sports, Science and Technology, Japan. TEM observation was carried out by using a facility in the Research Center for Ultra-High Voltage Electron Microscopy, Osaka University.

\section{References}

[1] P. Moine, J. Allain, B. Renker, J. Phy. F, 14, 2517 (1984)

[2] S. K. Satija, S. M. Shapiro, M. B. Salamon and C. M. Wayman Phys.Rev. B29, 6031(1984)

[3] M. B. Salamon, M. E. Meichle and C. M. Wayman, Phys. Rev. B 31, 7306 (1985)

[4] S. M. Shapiro, Y. Noda, Y. Fujii and Y. Yamada, Phys. Rev. B 30, 4314 (1984)

[5] Y. Yamada, Metal.Trans. A 19A, 777 (1988)

[6] Y. Murakami and D. Shindo, Philos. Mag. Lett 81, 9 (2001)

[7] C. M. Hawang, M. Meichle, M. B. Salamon and C. M. Wayman, Philos. Mag. A47, 9, 31 (1983)

[8] M.-S. Choi, T. Fukuda, T. Kakeshita, H. Mori, Philos.Mag. 86, 67 (2006)

[9] T. Yamamoto, T. Fukuda and T. Kakeshita: Mater. Trans. 47, 594 (2006) 